\title{
IDENTIFICACIÓN FORENSE DE LOCUTORES (I.F.L.): CATEGORIZACIÓN DE PARÁMETROS ACÚSTICOS Y FONO-ARTICULATORIOS DEL ESPAÑOL
}

\author{
Carlos Delgado Romero*, Maa Del Carmen Márquez Funes*,

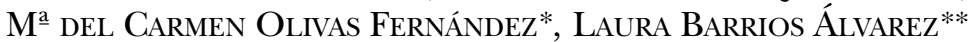 \\ *Laboratorio de Acústica Forense, C.G.P. Científica, D. G. Policía, Madrid \\ **C.T.I., Consejo Superior de Investigaciones Científicas, Madrid
}

\section{RESUMEN}

Todo proceso de identificación biométrica debe establecerse sobre unos parámetros de estudio convenientemente jerarquizados. Sin embargo, hasta el momento actual, la literatura científica del ámbito I.F.L. no aporta referencias de categorización suficientemente significativas. Tal circunstancia, podría asociarse a diferentes causas: breve trayectoria histórica de las técnicas, carácter multidisciplinar de las aproximaciones de estudio relacionadas, etc. Transcurridos veinte años desde su creación, el laboratorio de Acústica Forense de la Policía Científica española, considera llegado el momento de materializar su propia experiencia en unas referencias concretas de investigación científica. En este sentido, la determinación del valor identificativo de diversos parámetros puede considerarse ya un objetivo alcanzado. En el presente trabajo, se expone un rango de clasificación de los principales parámetros fonoarticulatorios y acústicos del español hablado, dentro del marco metodológico desarrollado por el laboratorio de la Policía Científica de España. Para ello, se ha realizado una evaluación parcial de la casuística real analizada por sus científicos durante un período de 5 años, incluyendo un total de 119 locutores y 2.329 parámetros.

Palabras clave: Parámetro Gestalt, nivel de identificación, probabilidad verbal, operacionalización, muestreo intencional no probabilístico, marcador distintivo, grado de presencia, marcador de presencia, intervariabilidad, intravariabilidad.

\section{ABSTRACT}

Any process of biometric identification must be established on the basis of categorized parameters. However, scientific literature on Forensic Speaker Identification (F.S.I.) has provided so far no contribution concerning specific references of categorization. This factor could be associated to different causes: fledging background of techniques, multidisciplinary nature of related approaches, and the like. Twenty years after being set up, the Forensic Acoustic Laboratory of the Spanish Scientific Police considered the moment to turn its own experience into concrete references of scientific investigation. In this sense, the identification weight of different parameters can be mentioned as an achieved goal. In this paper, a classification range of the main phono-articulatory and acoustic parameters in spoken Spanish, within the methodological framework developed by the Spanish Forensic Lab, is introduced. 
For that purpose, an evaluation of real cases analyzed by our experts in a five years period has been undertaken, involving 119 speakers and 2,329 different parameters.

Key Words: Gestalt parameter, Level of identification, verbal probability, operationalization, intentional no-probabilistic sampling, distinctive mark, rank of presence, mark of presence, intervariability, intravariability.

\section{INTRODUCCIÓN}

Aún teniendo en cuenta la valiosa contribución que representan los sistemas de reconocimiento automático (S.R.A.), hoy por hoy las perspectivas de análisis tradicional por expertos, es decir, aquellas dirigidas al examen de parámetros acústico-sonográficos y fonético-lingüísticos, continúan proporcionando numerosas claves en los estudios forenses de tipificación del habla.

Los criterios de selección y evaluación de los mencionados parámetros (que también podemos denominar parámetros Gestalt, pues se evalúan a través de inputs perceptivo-instrumentales) varían en función de la metodología I.F.L. utilizada y, fundamentalmente, de la lengua objeto de estudio. No obstante, si bien los aspectos relativos al método no representan una diferencia sustantiva a la hora de considerar un tipo de parámetro u otro (exceptuando el caso de los entornos S.R.A.), la estimación de los mismos en cuanto a su poder identificativo está claramente determinada por las características fonético-lingüísticas del habla analizada.

En la bibliografía relacionada con nuestro campo de trabajo no se encuentran referencias que de forma explícita y argumentada, identifiquen la mayor o menor relevancia identificativa de los diferentes rasgos Gestalt que caracterizan el habla. Simplemente, en algunos casos, se señalan o describen (Battaner y otros 2003, Black y otros 1973, Bolt y otros 1970, Clifford 1980, Delgado 2001, Dommelen 1997, Elaad y otros 1998, Endress y otros 1971, Gray y otros 1944, Hall 1975, Hartmann 1979, Hazen 1973, Helfrich 1979, Hollien 1990, Houlihan 1979, Kersta 1962, Koenig 1986, Köster 1981, Koval 2000, Künzel 1987, Ladefodged 1980, Marrero 2003, McGlone 1963, Morris 1998, Neiman 1990, Nolan 1990 y 1991, Pollack 1954, Potter 1947, Quilis 1981, Reich 1979, Rose 2002 , Rothman 1997, Suzuki 1997, Tosi 1972 y 1978], y en otros se enfatizan (Braun 1995, Delgado 2001, Doherty 1976, Koenig 1991, Koval 1999, Kuwabara 1995, Popov 1996, Smrkovski 1976, Stevens 1971, Tosi 1979). En el caso concreto de la lengua española, no existen precedentes a los estudios desarrollados por el laboratorio de Acústica Forense de la Policía Científica de España. Por esta razón y, como punto de partida a esta investigación, la información proporcionada por la casuística acumulada en dicho laboratorio ha sido considerada de alto interés. 
El objetivo fundamental del presente trabajo se centrará en reseñar y categorizar una serie de parámetros objeto de comparación en estudios periciales identificativos en lengua española que, desde el enfoque de la Fonética-Acústica y a través de la metodología I.F.L. practicada en nuestro laboratorio (Delgado 2001), pueden constituirse en referencias individualizadoras de máximo nivel. Lógicamente, quedarán excluidas aquellas características fuera del contexto de análisis forense convencional, frecuentemente circunscrito a las restricciones de información propias de la transferencia telefónica y condiciones de registro adversas. Por otra parte, aquellos parámetros no relacionados directamente con las características de resonancia (referidos a la fuente, lingüísticos, patológicos, etc.) también son considerados a pesar de estar sujetos a un mayor índice de variabilidad, pues siempre aportan una utilidad complementaria y, en contextos concretos, pueden resultar especialmente trascendentes.

\section{TRATAMIENTO DE LA INFORMACIÓN}

Ante la ausencia de referentes que de alguna manera vinculen los rasgos fono-articulatorios y acústicos con un determinado peso identificativo, la hipótesis que argumenta el presente trabajo se basa en asociar el valor de dichos rasgos a la utilidad que los mismos representan en las tareas de identificación del habla desarrolladas por los expertos. Es decir, presuponer que aquellos parámetros que más aparezcan en los estudios periciales de resultados más concluyentes, poseerán en principio un mayor poder individualizador. Lógicamente, si sólo se tuviese en cuenta el aspecto cuantitativo, las características del habla muy peculiares o infrecuentes y, por tanto, con alto valor identificativo (patológicas, disfuncionales, etc.) serían erróneamente ignoradas. Para evitar este tipo de error, el presente estudio ha combinado diferentes técnicas estadísticas que también incluyen la apreciación desde un enfoque cualitativo.

Cuatro cuestiones básicas constituyen el punto de partida:

- ¿Qué muestra es la adecuada para que el estudio resulte suficientemente representativo?

- ¿Qué variables pueden considerase relevantes y en qué medida intervienen?

- ¿Qué relaciones existen entre esas variables?

- ¿Qué criterio o criterios seguiremos a la hora de evaluar dichas variables?

Con el objeto de que las conclusiones del estudio queden enmarcadas en la realidad del mundo forense, la muestra objeto de análisis ha 
sido seleccionada de entre los casos reales archivados en el laboratorio de Acústica de la Policía, concretamente, los comprendidos en el período 1998-2002. En dicho período, la metodología I.F.L. desarrollada en el laboratorio se encontraba ya sólidamente consolidada y los científicos noveles contaban con un mínimo de dos años de experiencia en prácticas periciales.

Solamente han sido seleccionados para el estudio aquellos informes de experto que concluían en un nivel de decisión de la máxima certeza, el denominado «nivel de identificación». A este respecto, es conveniente aclarar que la fórmula de decisión utilizada en el laboratorio de la Policía Científica viene representada por una «escala cualitativa» en la que se definen diferentes niveles de certeza (Delgado 2001). Cuando los distintos sistemas de análisis que integran el método indican máximos niveles de similitud entre las muestras comparadas, el nivel de conclusión se define como «nivel de identificación». Esta expresión no ha de equipararse a una identificación positiva (100\%) sino al máximo nivel de certeza que un determinado experto con una formación, experiencia y metodología concretas, puede llegar a obtener. Se ha utilizado, por tanto, un muestreo intencional no probabilístico. Este tipo de muestreo se caracteriza por un esfuerzo deliberado para obtener una muestra representativa mediante la inclusión en ella de objetos típicos.

El número de informes I.F.L. analizados ha sido de 119, de los cuales, 98 correspondían a locutores varones y 21 a informantes mujeres. Todo ello reportó un total de 2.329 registros de características.

Han sido establecidas cuatro variables básicas: «unidad», "peculiaridad», «medición» y «valoración». La apreciación conjunta de las mismas define una referencia suficientemente significativa que, en adelante, será denominada «marcador distintivo».

Una vez definidas las variables, se han adoptado los procesos considerados más adecuados para la codificación, transformación y representación cuantitativa de las mismas, así como para los consiguientes análisis.

\section{DESCRIPCIÓN DE PARÁMETROS INDICADORES}

Tras una exploración previa de la casuística seleccionada se definen cuatro variables: unidad, peculiaridad, valoración y medición.

1. Unidad: elemento de comparación analizado.

2. Peculiaridad: las variaciones que la unidad presenta y que se manifiestan a través de correlatos acústicos y articulatorios. Ejemplos: sonoridad, tonalidad, intensidad, cantidad. Además se incluyen otras variaciones adicionales (realizaciones monofonemáticas, realización fonética cero...). 
3. Valoración: apreciación que de una peculiaridad, unidad o medición hace el perito en su análisis comparativo (relevante, adelantado, retrasado...).

4. Medición: representa el número de estimaciones efectuadas. Puede tratarse tanto del número de peculiaridades medidas como de cualquier otra de medida realizada durante el proceso de comparación.

Estas variables, representadas como «unicod», «pecucod», «valoracod» $\mathrm{y}$ «forymed», han sido codificadas tal como queda descrito en la siguiente tabla:

\begin{tabular}{|c|c|c|}
\hline ID & Unidad & Unicod \\
\hline 1 & Unidad cero & 0 \\
\hline 2 & $/ \mathrm{a} /$ & $\mathrm{a}$ \\
\hline 3 & $/ \mathrm{b} /$ & $\mathrm{b}$ \\
\hline 4 & $/ \mathrm{B} /$ & $\mathrm{BM}$ \\
\hline 5 & $/ \mathrm{d} /$ & $\mathrm{d}$ \\
\hline 6 & $/ \mathrm{D} /$ & $\mathrm{DM}$ \\
\hline 7 & $/ \mathrm{e} /$ & $\mathrm{e}$ \\
\hline 8 & $/ \mathrm{f} /$ & $\mathrm{f}$ \\
\hline 9 & $/ \mathrm{g} /$ & $\mathrm{g}$ \\
\hline 10 & $/ \mathrm{G} /$ & $\mathrm{GM}$ \\
\hline 11 & $/ \mathrm{i} /$ & $\mathrm{i}$ \\
\hline 12 & $/ \mathrm{k} /$ & $\mathrm{k}$ \\
\hline 13 & $/ \mathrm{l} /$ & $\mathrm{l}$ \\
\hline 14 & $/ \lambda /$ & $\mathrm{ll}$ \\
\hline 15 & $/ \mathrm{m} /$ & $\mathrm{m}$ \\
\hline 16 & $/ \mathrm{n} /$ & $\mathrm{n}$ \\
\hline 17 & $/ \mathrm{n} /$ & $\tilde{\mathrm{n}}$ \\
\hline 18 & $/ \mathrm{N} /$ & $\mathrm{NM}$ \\
\hline & & \\
\hline
\end{tabular}

\begin{tabular}{|r|l|c|}
\hline ID & \multicolumn{1}{|c|}{ Peculiaridad } & Pecucod \\
\hline 1 & Acumulación de energía & ae \\
\hline 2 & $\begin{array}{l}\text { Acumulación energía } \\
\text { armónica }\end{array}$ & aea \\
\hline 3 & Altura formántica & af \\
\hline 4 & Africada & afr \\
\hline 5 & Asimilación & asi \\
\hline 6 & Aspirada (realización) & asp \\
\hline 7 & 2 barras & ba2 \\
\hline 8 & Barra & bar \\
\hline 9 & Barra energía difusa & bed \\
\hline 10 & Barra oclusivo/fricativo & bof \\
\hline 11 & Voz dicrótica & dic \\
\hline 12 & Duración oclusión & doc \\
\hline 13 & Duración & dur \\
\hline 14 & $\begin{array}{l}\text { Estridencias } \\
\text { (no máximas) }\end{array}$ & est \\
\hline 15 & Esvarabático & esv \\
\hline 16 & Fonética cero & f0 \\
\hline 17 & Fricativa (realización) & fri \\
\hline 18 & Imprecisa (realización) & imp \\
\hline
\end{tabular}

\begin{tabular}{|c|l|c|}
\hline ID & Tipo de Valoración & Valoracod \\
\hline 1 & Sin valorar. & 0 \\
\hline 2 & $\begin{array}{l}\text { abierto/ } \\
\text { adelantado }\end{array}$ & aad \\
\hline 3 & adelantado & ade \\
\hline 4 & Asibilada & asb \\
\hline 5 & F1 intensidad & fl \\
\hline 6 & Hipernasalización & hpe \\
\hline 7 & Hiponasalización & hpo \\
\hline 8 & Locución lenta & len \\
\hline 9 & Voz monótona & mon \\
\hline 10 & Relevante ae & rae \\
\hline 11 & Locución rápida & rap \\
\hline 12 & Relevante barra & rba \\
\hline 13 & Relevante duración & rdu \\
\hline 14 & Relevante aea & rea \\
\hline 15 & $\begin{array}{l}\text { Relevante } \\
\text { esvarabático }\end{array}$ & res \\
\hline 16 & retrasado & ret \\
\hline 17 & Ratio locutivo & rlo \\
\hline 18 & $\begin{array}{l}\text { Relevante mom. } \\
\text { Oclusivo }\end{array}$ & roc \\
\hline
\end{tabular}




\begin{tabular}{|c|c|c|c|c|c|c|c|c|}
\hline \multirow{2}{*}{$\frac{1}{1}$} & & & \multicolumn{2}{|c|}{$\vdash$} & \multirow[b]{2}{*}{ rre } \\
\hline & /0/ & 0 & 19 & Intensidad & int & 19 & Relevante relajada & \\
\hline 20 & $/ \mathrm{p} /$ & $\mathrm{p}$ & 20 & Locución & loc & 20 & Relevante tensa & rte \\
\hline 21 & $/ \mathrm{f} /$ & r & 21 & Máximas estridencias & mes & 21 & Relevante VOT & rvo \\
\hline 22 & $/ \mathrm{R} /$ & RM & 22 & Nasalidad & nas & 22 & F2, F3 solapados & s23 \\
\hline 23 & $/ \mathrm{r} /$ & $\mathrm{rr}$ & 23 & Oclusiva (realización & ocl & 23 & Locución similar & $\operatorname{sim}$ \\
\hline 24 & $/ \mathrm{s} /$ & $\mathrm{s}$ & 24 & $\begin{array}{l}\text { Oclusiva-fricativa- } \\
\text { oclusiva }\end{array}$ & ofo & 24 & Spike & spi \\
\hline 25 & sec. cons. & $\mathrm{sc}$ & 25 & Oclusión = fricación & ogf & 25 & $\begin{array}{l}\text { Suprasegmento } \\
\text { prosódico }\end{array}$ & $\sup$ \\
\hline 26 & sec. mixta & $\mathrm{sm}$ & 26 & Oclusión > fricación & omf & & & \\
\hline 27 & sec. voc. & sv & 27 & Oclusión < fricación & onf & & & \\
\hline 28 & $/ \mathrm{t} /$ & $\mathrm{t}$ & 28 & $\begin{array}{l}\text { Palatalizada } \\
\text { (realización) }\end{array}$ & $\mathrm{pal}$ & & & \\
\hline 29 & $/ \mathrm{t} /$ & ts & 29 & $\begin{array}{l}\text { Reducción (monof., } \\
\text { bifonem, etc...) }\end{array}$ & red & ID & Tipo de Medición & Forymed \\
\hline 30 & $/ \mathrm{u} /$ & $\mathrm{u}$ & 30 & Relajada & rel & 0 & Sin mediciones & 0 \\
\hline 31 & $/ \mathrm{X} /$ & $\mathrm{j}$ & 31 & Respiración alterada & res & 1 & Una medición & 1 \\
\hline 32 & $/ \hat{\mathrm{\jmath}} /$ & y & 32 & Sonorizada (realización) & son & 2 & Dos mediciones & 2 \\
\hline 33 & $/ \theta /$ & $\mathrm{z}$ & 33 & Sorda (realización) & sor & 3 & Tres mediciones & 3 \\
\hline & & & 34 & Sustitución & sus & 4 & Cuatro mediciones & 4 \\
\hline & & & 35 & Tensa & ten & 5 & $\begin{array}{l}\text { Más de } 4 \\
\text { mediciones }\end{array}$ & 5 \\
\hline & & & 36 & Transición & tra & & & \\
\hline & & & 37 & Velarizada (realización) & vel & & & \\
\hline & & & 38 & Vocalizada (realización) & voc & & & \\
\hline & & & 39 & $\begin{array}{l}\text { VOT (y momento } \\
\text { plosivo) }\end{array}$ & vot & & & \\
\hline
\end{tabular}

Tabla explicativa de códigos de las variables.

Una vez ordenados los datos para hacerlos accesibles a cualquier técnica estadística, se realiza un examen gráfico de la «naturaleza de las variables individuales» y un análisis descriptivo univariante que permite cuantificar algunos aspectos de tales datos. Si bien los resultados apor- 
tan una información descriptiva interesante, se hace necesaria otra perspectiva de estudio que explique las relaciones entre dichas variables. De dicho análisis cabe destacar los diferentes valores de frecuencia:

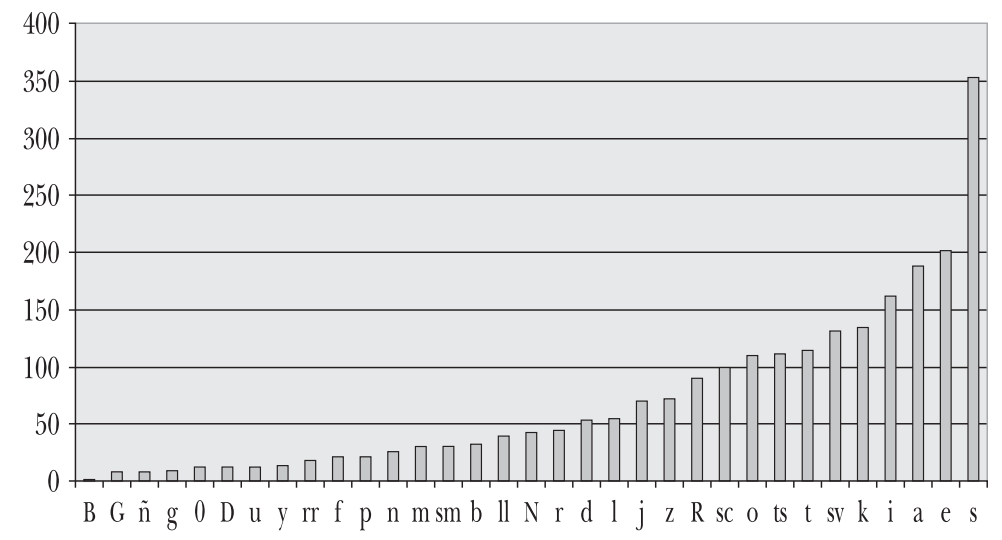

CuAdro 1: Tabla de frecuencia de la variable «Unidades»(UNICOD).

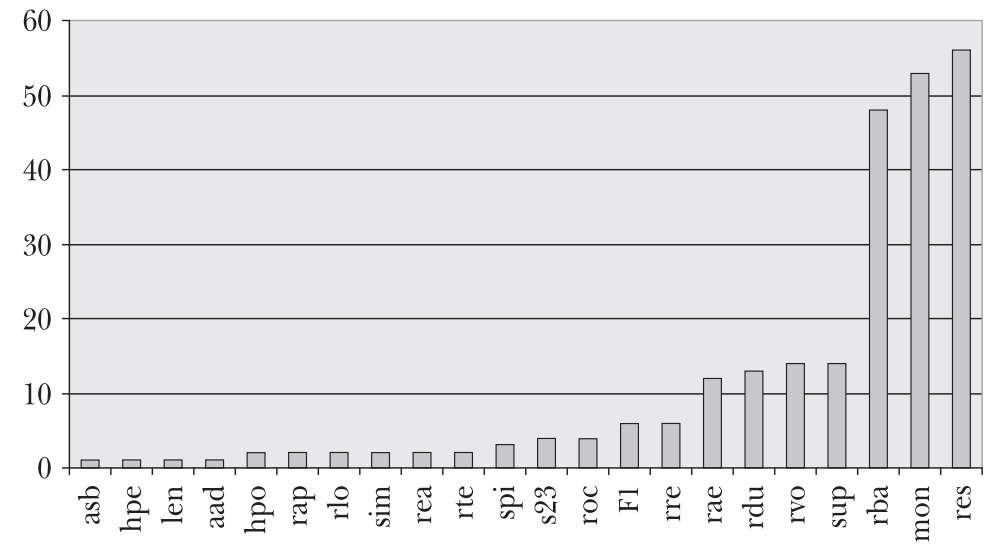

CUAdro 2: Tabla de frecuencia de la variable «Valoración» (VALORACOD). Quedan excluidas las referencias superiores a 200 estimaciones, que se representan en la tabla siguiente. 


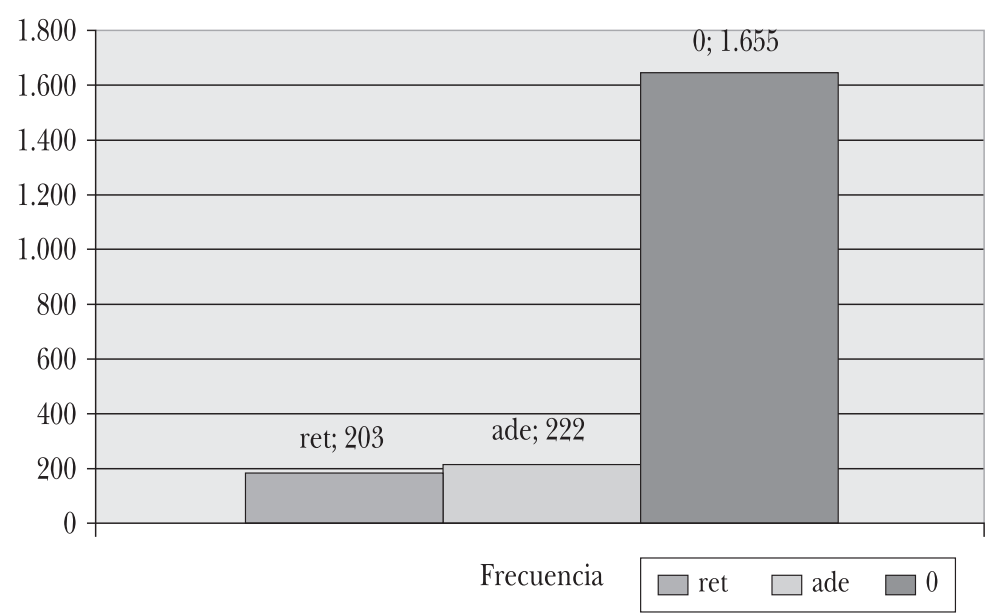

CUADro 3: Tabla de frecuencia de la variable «Valoración» (VALORACOD), en aquellos casos que superan las 200 estimaciones.

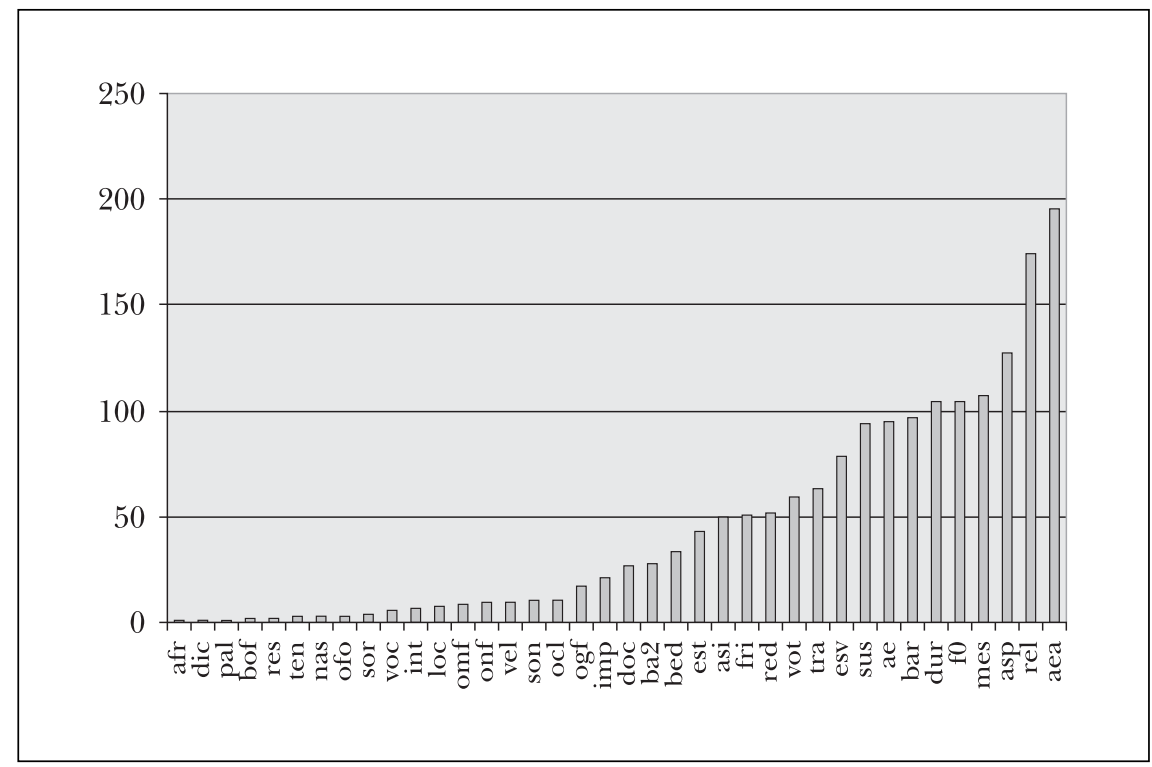

Cundro 4: Tabla de frecuencia de la variable «Peculiaridad» (PECUCOD).

La peculiaridad «af» por alcanzar una frecuencia de 616 no ha sido incluida. 


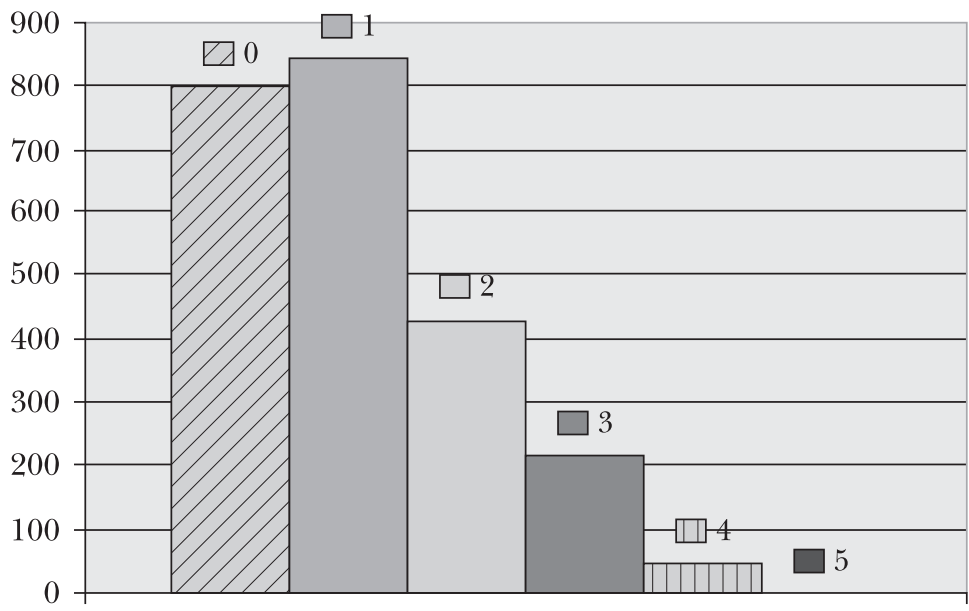

CuAdro 5: Tabla de frecuencia de la variable «Mediciones» (FORYMED).

\section{RELACIONES Y CLASIFICACIÓN DE PARÁMETROS}

Una vez observados de forma independiente los datos cuantitativos de las diferentes variables, resulta necesario observar su comportamiento de forma interrelacionada. Esto se hace mediante un análisis de componentes principales categóricos (CatPCA) por escalamiento óptimo (Gifi A. 1990).

De los resultados obtenidos con el análisis de componentes principales, cabe destacar:

- Una fuerte correlación positiva entre:

- unicod y pecucod,

- unicod y forymed,

- forymed y valoracod.

- Una correlación negativa entre:

- valoracod con unicod,

- valoracod con pecucod,

- forymed con pecucod. 
Saturaciones en las componentes

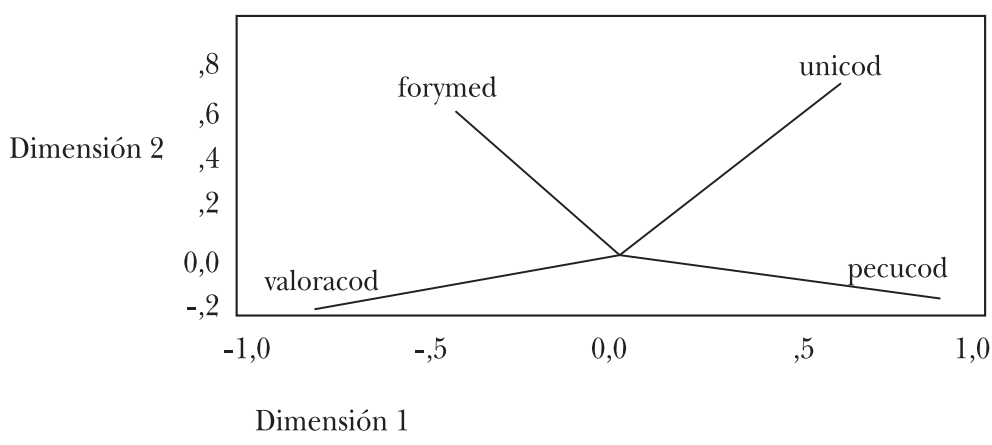

Normalización principal por variable

CuAdro 6: Análisis interrelacionado de componentes. Los ángulos $<90^{\circ}$ determinan una correlación positiva. Los $>90^{\circ}$ indican una correlación negativa.

De todo ello puede deducirse la existencia de una relación entre las variables, si bien no son linealmente dependientes.

El siguiente paso consiste en encontrar patrones entre los objetos que tengan un comportamiento similar en estas cuatro variables estudiadas. Se determina mediante cuatro análisis cluster de k-medias. (Hartigan 1975).

La pretensión es identificar un número pequeño de grupos por cada variable analizada, de modo que los objetos pertenecientes a un mismo grupo sean muy semejantes entre sí y los objetos pertenecientes a grupos diferentes muestren un comportamiento distinto.

Por tanto, el objetivo de la clasificación es encontrar grupos de casos de las distintas variables (unidad, peculiaridad, valoración y medición) que puedan aportar una relevancia significativa al marcador distintivo. Para llevar a cabo esta tarea, se hace precisa una transformación cuantitativa que facilite cualquier labor estadística posterior.

En definitiva, el proceso busca obtener los referentes empíricos de las variables, es decir, reducir el nivel de abstracción de la variable a indicadores que puedan medirse mediante índices o indagarse mediante ítems de los instrumentos de recopilación de información. 


\subsection{Proceso de tratamiento y transformación de datos}

Una vez definidas las variables, han de especificarse los atributos mensurables para la cuantificación de las mismas:

- La variable «Unidad»:

» Frecuencia de aparición de cada unidad

» Frecuencia de aparición de las peculiaridades para cada unidad

» Frecuencia de aparición de las mediciones para cada unidad

» Frecuencia de aparición de las valoraciones para cada unidad

- La variable «Peculiaridad»:

» Frecuencia de aparición de cada peculiaridad

» Frecuencia de aparición de las unidades para cada peculiaridad

» Frecuencia de aparición de las mediciones para cada peculiaridad

» Frecuencia de aparición de las valoraciones para cada peculiaridad

- La variable «Medición»:

» Frecuencia de aparición de la medición

» Frecuencia de aparición de las peculiaridades para cada medición

» Frecuencia de aparición de las unidades para cada medición

» Frecuencia de aparición de las valoraciones para cada medición

- La variable «Valoración»:

» Frecuencia de aparición de la valoración

» Frecuencia de aparición de la peculiaridad en cada valoración

» Frecuencia de mediciones en cada valoración

» Frecuencia de unidad en cada valoración

\subsubsection{Fases del proceso}

- En primer lugar se realiza el cálculo de frecuencias de aparición de cada respuesta posible dentro de las distintas variables. Efectuados dichos cálculos, definimos cuatro nuevas tablas: «f_uni», «f_pecu», «f_val»y «frecufym».

- Se procede a la transformación cuantitativa de las variables respecto de sus componentes, en atención a esos valores de frecuencia. 
- Se realiza un cálculo de probabilidad para cada una de las respuestas obtenidas.

- A continuación se realiza una «estandarización» de los resultados de probabilidad obtenidos y se determina una probabilidad final para cada valor de la variable.

\subsubsection{Procedimiento de estandarización}

Como paso previo se divide cada valor de probabilidad por el máximo alcanzado en la variable analizada.

Posteriormente, se realiza la suma de los valores de probabilidad estandarizados de los atributos, y como resultado de la transformación cuantitativa aplicada, se obtienen cuatro nuevas variables: «valor_uni», «valor_pecu», «valor_val»y «valor_fym».

De esta forma, ya es posible obtener la representación de las variables objeto de estudio con valores numéricos uniformes y estandarizados y, por tanto, realizar el análisis de la información con métodos cuantitativos.

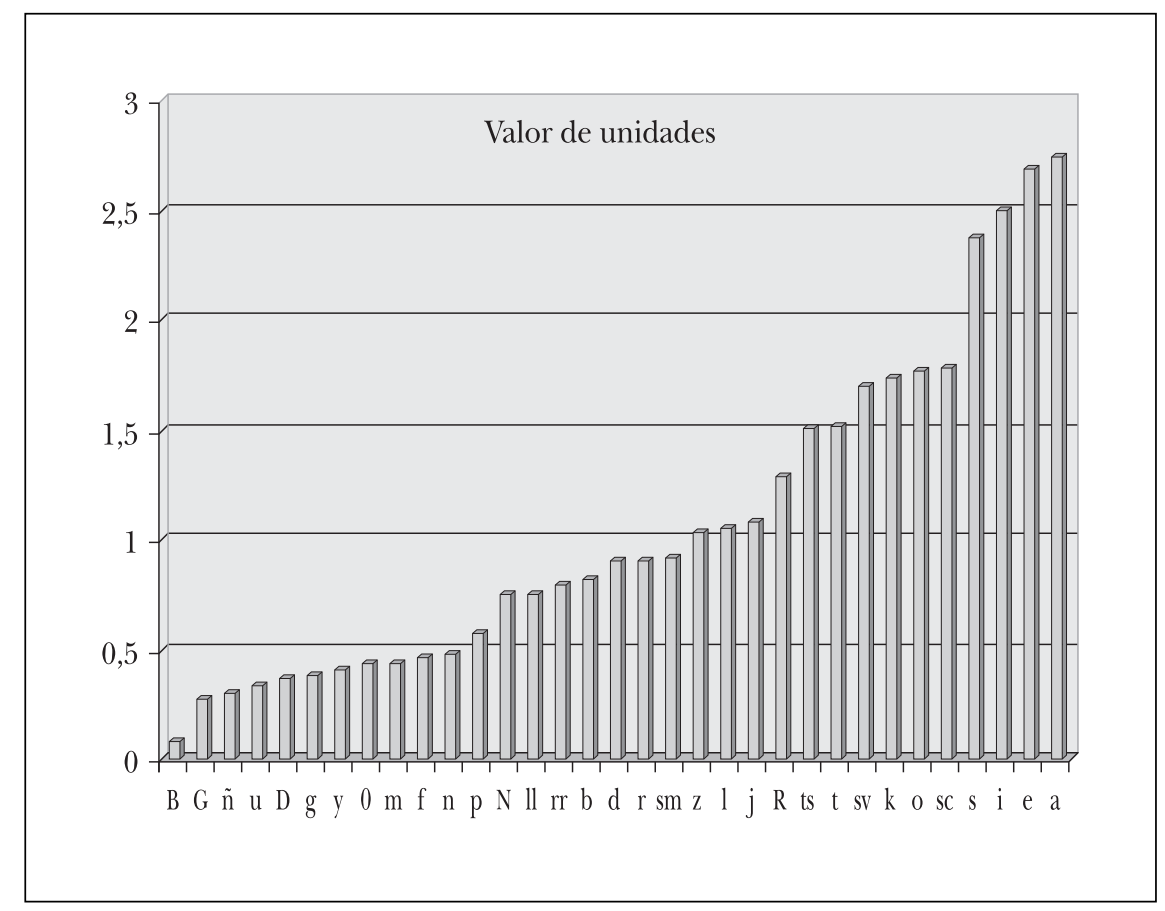

CUADro 7: Valor de Unidades, «VALOR_UNI». 


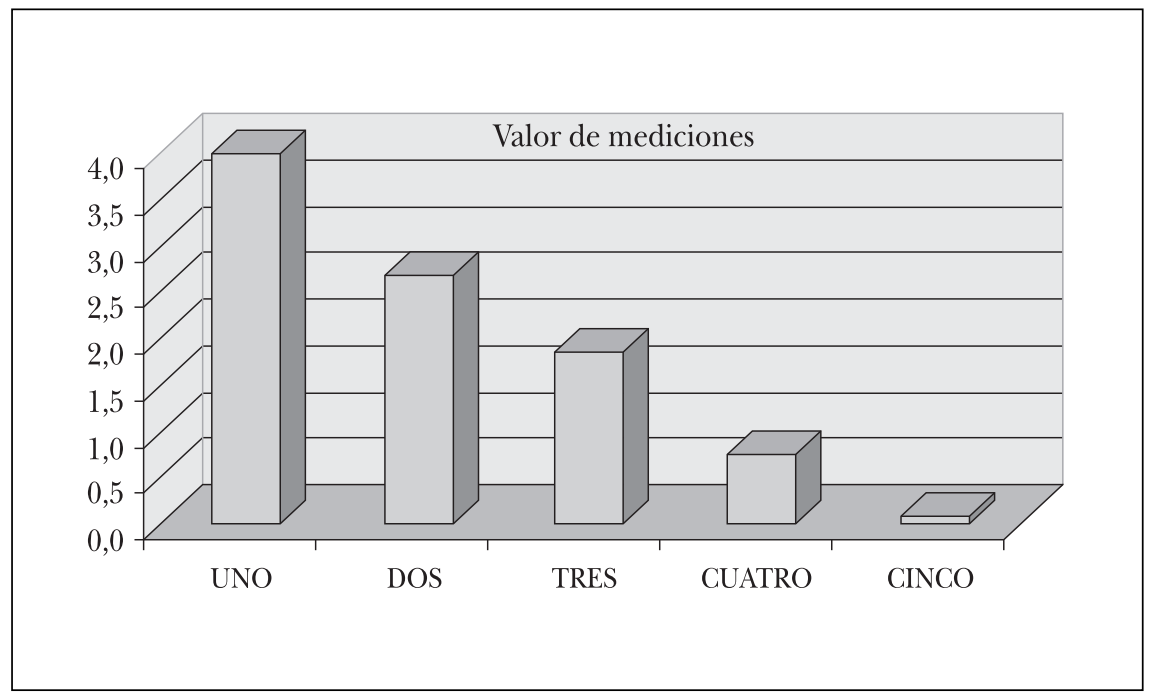

CUADro 8: Valor de Mediciones, «VALOR_FYM».

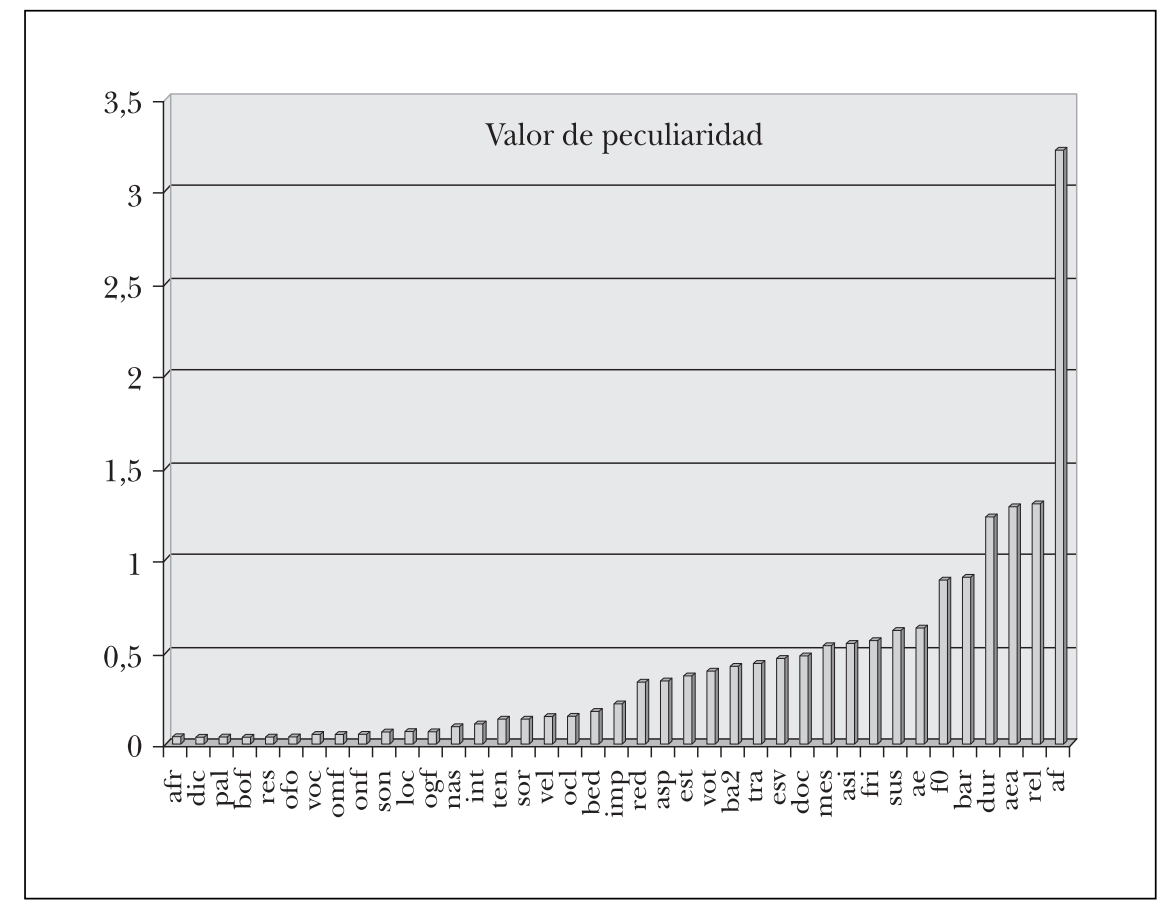

CuAdro 9: Valor de Peculiaridad, «VALOR_PECU». 


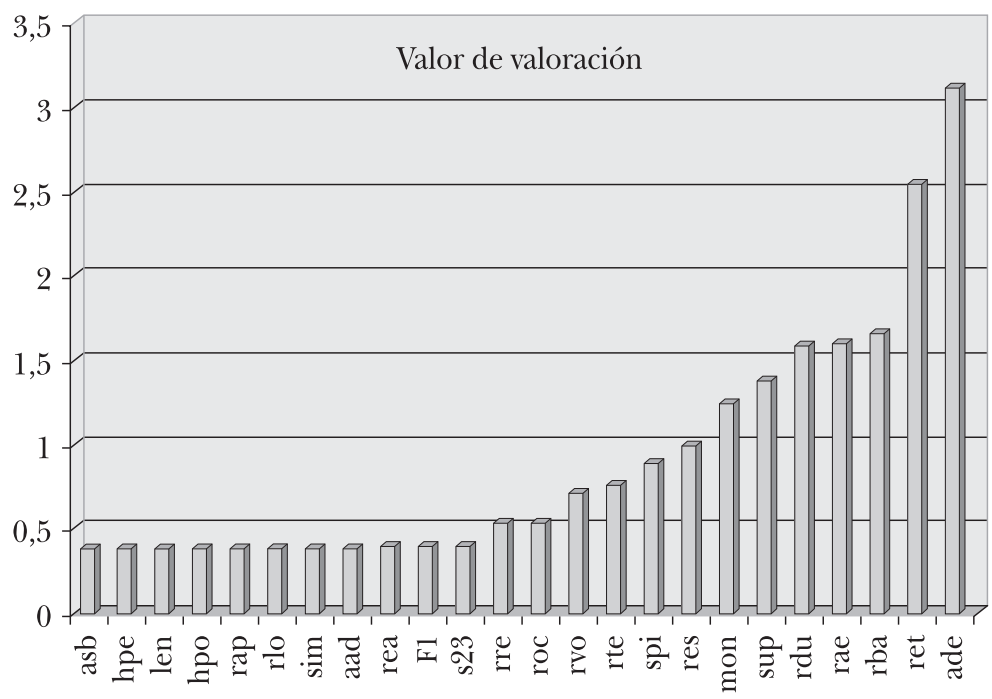

CUADro 10: Valor de Valoración, «VALOR_VAL».

El procesado de datos y los sucesivos análisis estadísticos han sido realizados con el programa SPSS 15.0.

\section{Patrones Significativos}

Como se ha descrito en el apartado anterior, una vez analizadas en modo cuantitativo las variables expresadas y sus relaciones, se efectúa un estudio conjunto de las mismas. Mediante análisis cluster se obtienen también cuatro grupos diferenciados, distintos para cada variable: QCLU, QCLP, QCLY y QCLV. A cada grupo o cluster se le confiere un "grado de presencia», otorgando el grado (A) al grupo de datos cuyo valor estandarizado es mayor, y el grado (D) al de menor nivel. Entre dichos valores se situarían, en orden de mayor a menor, los grados (B) y (C).

En la siguiente tabla, los diferentes grados de presencia se representan como las variables QU, QP, QY y QV. Con la concatenación de estos valores, se obtiene la variable «mdabcd» (marcador distintivo). Esto es lo que finalmente representa una observación conjunta. 


\begin{tabular}{|c|c|c|c|c|c|c|c|c|c|c|c|c|c|c|c|}
\hline HABLA & EXPTE & SEXO & CODIU & CODIP & FYM & CODIV & QCLU & QCLP & QCLY & QCLV & QU & QP & QY & QV & mdabcd \\
\hline 1 & $10 / 01-1 \mathrm{~V}$ & 1 & 22 & 5 & 0 & 1 & 2 & 2 & 1 & 1 & C & C & $\mathrm{D}$ & D & C,C,D,D \\
\hline 1 & $10 / 01-1 \mathrm{~V}$ & 1 & 13 & 2 & 5 & 1 & 2 & 3 & 1 & 1 & C & B & D & D & C,B,D,D \\
\hline 1 & $10 / 01-1 \mathrm{~V}$ & 1 & 16 & 2 & 3 & 1 & 3 & 3 & 2 & 1 & D & B & $\mathrm{C}$ & D & D,B,C,D \\
\hline 1 & $10 / 01-1 \mathrm{~V}$ & 1 & 24 & 5 & 0 & 1 & 4 & 2 & 1 & 1 & A & C & D & D & $\mathrm{A}, \mathrm{C}, \mathrm{D}, \mathrm{D}$ \\
\hline 1 & $10 / 01-1 \mathrm{~V}$ & 1 & 28 & 9 & 0 & 12 & 1 & 1 & 1 & 2 & B & D & D & B & $\mathrm{B}, \mathrm{D}, \mathrm{D}, \mathrm{B}$ \\
\hline 1 & $10 / 01-1 \mathrm{~V}$ & 1 & 28 & 9 & 0 & 12 & 1 & 1 & 1 & 2 & B & D & D & B & $\mathrm{B}, \mathrm{D}, \mathrm{D}, \mathrm{B}$ \\
\hline 1 & $10 / 01-1 \mathrm{~V}$ & 1 & 28 & 9 & 0 & 12 & 1 & 1 & 1 & 2 & B & D & D & B & $\mathrm{B}, \mathrm{D}, \mathrm{D}, \mathrm{B}$ \\
\hline 1 & $10 / 01-1 \mathrm{~V}$ & 1 & 28 & 9 & 0 & 12 & 1 & 1 & 1 & 2 & B & D & D & B & B,D,D,B \\
\hline 1 & $10 / 01-1 \mathrm{~V}$ & 1 & 28 & 9 & 0 & 12 & 1 & 1 & 1 & 2 & B & D & D & B & $\mathrm{B}, \mathrm{D}, \mathrm{D}, \mathrm{B}$ \\
\hline 1 & $10 / 01-1 \mathrm{~V}$ & 1 & 28 & 9 & 0 & 12 & 1 & 1 & 1 & 2 & B & D & D & B & B,D,D,B \\
\hline 1 & $10 / 01-1 \mathrm{~V}$ & 1 & 28 & 9 & 0 & 12 & 1 & 1 & 1 & 2 & B & D & D & B & $\mathrm{B}, \mathrm{D}, \mathrm{D}, \mathrm{B}$ \\
\hline 1 & $10 / 01-1 \mathrm{~V}$ & 1 & 22 & 2 & 3 & 1 & 2 & 3 & 2 & 1 & C & B & $\mathrm{C}$ & D & $\mathrm{C}, \mathrm{B}, \mathrm{C}, \mathrm{D}$ \\
\hline 1 & $10 / 01-1 \mathrm{~V}$ & 1 & 27 & 29 & 2 & 9 & 1 & 1 & 3 & 3 & B & D & B & C & $\mathrm{B}, \mathrm{D}, \mathrm{B}, \mathrm{C}$ \\
\hline 1 & $10 / 01-1 \mathrm{~V}$ & 1 & 24 & 21 & 1 & 1 & 4 & 2 & 4 & 1 & A & C & A & D & $\mathrm{A}, \mathrm{C}, \mathrm{A}, \mathrm{D}$ \\
\hline 1 & $10 / 01-1 \mathrm{~V}$ & 1 & 24 & 21 & 1 & 1 & 4 & 2 & 4 & 1 & A & C & A & D & $\mathrm{A}, \mathrm{C}, \mathrm{A}, \mathrm{D}$ \\
\hline 1 & $10 / 01-1 \mathrm{~V}$ & 1 & 7 & 3 & 3 & 1 & 4 & 4 & 2 & 1 & A & A & $\mathrm{C}$ & D & $\mathrm{A}, \mathrm{A}, \mathrm{C}, \mathrm{D}$ \\
\hline 1 & $10 / 01-1 \mathrm{~V}$ & 1 & 7 & 3 & 3 & 1 & 4 & 4 & 2 & 1 & A & A & $\mathrm{C}$ & D & $\mathrm{A}, \mathrm{A}, \mathrm{C}, \mathrm{D}$ \\
\hline 1 & $10 / 01-1 \mathrm{~V}$ & 1 & 7 & 3 & 3 & 3 & 4 & 4 & 2 & 4 & A & A & C & A & $\mathrm{A}, \mathrm{A}, \mathrm{C}, \mathrm{A}$ \\
\hline 1 & $10 / 01-1 \mathrm{~V}$ & 1 & 7 & 3 & 3 & 3 & 4 & 4 & 2 & 4 & A & A & $\mathrm{C}$ & A & $\mathrm{A}, \mathrm{A}, \mathrm{C}, \mathrm{A}$ \\
\hline 1 & $10 / 01-1 \mathrm{~V}$ & 1 & 7 & 3 & 3 & 3 & 4 & 4 & 2 & 4 & A & A & C & A & $\mathrm{A}, \mathrm{A}, \mathrm{C}, \mathrm{A}$ \\
\hline 1 & $10 / 01-1 \mathrm{~V}$ & 1 & 11 & 3 & 1 & 3 & 4 & 4 & 4 & 4 & A & A & A & A & $\mathrm{A}, \mathrm{A}, \mathrm{A}, \mathrm{A}$ \\
\hline 1 & $10 / 01-1 \mathrm{~V}$ & 1 & 11 & 3 & 1 & 3 & 4 & 4 & 4 & 4 & A & A & A & A & $\mathrm{A}, \mathrm{A}, \mathrm{A}, \mathrm{A}$ \\
\hline 1 & $10 / 01-1 \mathrm{~V}$ & 1 & 11 & 3 & 1 & 3 & 4 & 4 & 4 & 4 & A & A & A & A & $\mathrm{A}, \mathrm{A}, \mathrm{A}, \mathrm{A}$ \\
\hline 1 & $10 / 01-1 \mathrm{~V}$ & 1 & 2 & 3 & 1 & 3 & 4 & 4 & 4 & 4 & A & A & A & A & $\mathrm{A}, \mathrm{A}, \mathrm{A}, \mathrm{A}$ \\
\hline
\end{tabular}

CuAdro 11: Se corresponde con la información codificada de las características del hablante $\mathrm{n}^{\mathrm{o}} 1$ estudiado en el expediente 10/01-1V. 
Para cada hablante, existe un número variable de marcadores distintivos identificados mediante ítems nominales con sentido cualitativo, registrados en las variables ya cuantificadas. Estos marcadores pueden repetirse en un mismo hablante.

Veamos a modo de ejemplo (fila resaltada en negrita en el cuadro anterior) el desglose de información codificada para la locución «tontería», realizada por el hablante $\mathrm{n}^{\underline{0}} 1$ :

- CODIU (16): se corresponde con la realización de «/n/».

- CODIP (2): se corresponde con «aea», acumulación de energía armónica.

- FYM (3): se corresponde con «3» mediciones.

- CODIV (1) indica «sin valoración»

Los cluster correspondientes a estos valores de variables serán:

- QCLU (3) al que asociamos la medida de presencia QU (D) se corresponde con CODIU «/n/».

- QCLP (3) al que asociamos la medida de presencia QP (B) se corresponde con CODIP «aea».

- QCLY (2) al que asociamos la medida de presencia QY (C) se corresponde con FYM «3».

- QCLV (1) al que asociamos la medida de presencia QV (D) se corresponde con CODIV «sin valoración».

- Se crea la variable «mdabcd» con la concatenación de estos valores así ordenados QU, QP, QY, QV: «D, B, C,D».

De esta forma se obtiene una perspectiva conjunta de las variables propuestas que facilita la ejecución de un posterior análisis.

\subsection{Marcador de presencia. Determinación del vector de presencia y su frecuencia ' $n$ '}

Partiendo de la variable obtenida «mdabcd», se ha de determinar la frecuencia de sus casos. Mediante segmentación de la Base de Datos por hablante, se halla a su vez, la frecuencia de los casos de dicha variable en cada locutor. Con estos estadísticos, se obtienen dos valores que indicarán, respectivamente, los factores de intervariabilidad («intermd») e intravariablidad («intramd») del marcador distintivo.

Una vez obtenidos estos datos puede configurarse un nuevo esquema. Sirva como ejemplo el de la información relativa al hablante $\mathrm{n}^{\mathrm{o}} 1$ : 


\begin{tabular}{|c|c|c|c|c|}
\hline Hablante & Exp & mdabcd & intramd & intermd \\
\hline 1 & $10 / 01-1 V$ & A, A, A, A & 4 & 180 \\
\hline 1 & $10 / 01-1 V$ & A, A, C, A & 3 & 61 \\
\hline 1 & $10 / 01-1 V$ & A, A, C, D & 2 & 45 \\
\hline 1 & $10 / 01-1 V$ & A, C,A,D & 2 & 84 \\
\hline 1 & $10 / 01-1 V$ & A, C, D, D & 1 & 101 \\
\hline 1 & $10 / 01-1 V$ & B, D, B, C & 1 & 35 \\
\hline 1 & $10 / 01-1 V$ & B, D, D, B & 7 & 13 \\
\hline 1 & $10 / 01-1 V$ & C, B, C,D & 1 & 20 \\
\hline 1 & $10 / 01-1 V$ & C, B, D, D & 1 & 86 \\
\hline 1 & $10 / 01-1 V$ & C, C, D, D & 1 & 145 \\
\hline 1 & $10 / 01-1 V$ & D, B, C, D & 1 & 1 \\
\hline
\end{tabular}

En el cuadro se observa cómo queda constituido el marcador de presencia, integrado por las variables «mdabcd», «intramd»e «intermd». De esta manera, toda la información relacionada con el marcador distintivo puede ser fácilmente apreciada.

Si se analiza uno de los casos (en negrita en la tabla) podrá apreciarse la relevancia de la información obtenida. Así por ejemplo, el marcador distintivo B, D, D, B al que corresponden el valor más alto para «intramd» (7) y un valor relativamente bajo en «intermd» (13), resultaría un elemento de alto peso identificativo para el perito, dado que se trata de una característica muy poco frecuente en la base de datos y sin embargo, muy frecuente en el hablante. Además, puede observarse que se trata de una característica con un valor alto en unidad (B) y valoración (B) mientras que en peculiaridad (D) y mediciones (D) es un valor bajo.

Los datos de este caso en particular se refieren a la articulación del fonema $/ t /$ en siete realizaciones, en las que se aprecia «barra configurada en energía difusa» (bed) «sin mediciones» y valorada por el perito como «relevante» (rba).

Vista la información que aportan las variables «intramd»e «intermd», se hace preciso establecer un nuevo estudio de las mismas («estudio de razón»: «intramd» respecto de «intermd») cuya finalidad será hallar un 
estadístico suficientemente significativo para relacionar los conceptos intravariabilidad e intervariabilidad.

El procedimiento estadístico de la razón proporciona una amplia lista de estadísticos de resumen para describir la razón entre dos variables. Los resultados obtenidos proporcionan un único dato por hablante expresado en tres conceptos diferentes. De ellos, el «coeficiente de dispersión» ha sido considerado el de más utilidad para los objetivos de nuestro estudio.

De esta manera, al final se obtiene un fichero que identifica al hablante con cada uno de sus marcadores diferenciados y establece una relación ordenada de los hablantes, en función de los resultados obtenidos tras el cálculo de los estadísticos de razón para el coeficiente de dispersión:

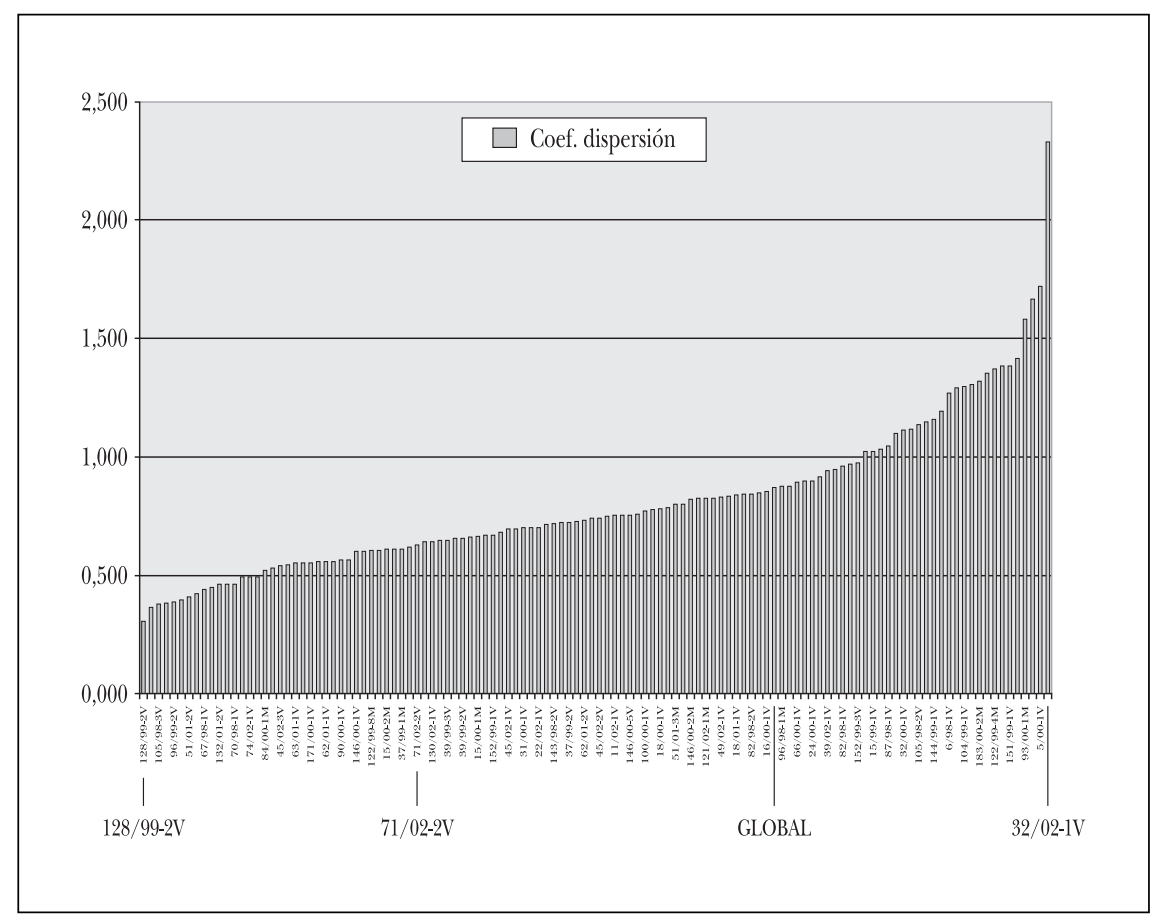

\section{ANÁLISIS DE CASOS SIGNIFICATIVOS}

Partiendo de la distribución de los datos expresada en el gráfico anterior, se centra el análisis en las referencias más extremas y otra relativamente próxima al valor global: 
1. Información resumida del expediente 32/02-1V correspondiente al Hablante $n^{-} 70$

\begin{tabular}{|c|c|c|c|c|}
\hline habla & $\exp$ & md & intramd & intermd \\
\hline 70 & $32 / 02-1 \mathrm{~V}$ & $\mathbf{A}, \mathbf{A}, \mathbf{B}, \mathbf{A}$ & 10 & 93 \\
\hline 70 & $32 / 02-1 \mathrm{~V}$ & $\mathbf{A}, \mathbf{C}, \mathbf{A}, \mathbf{D}$ & 6 & 84 \\
\hline 70 & $32 / 02-1 \mathrm{~V}$ & $\mathbf{A}, \mathbf{D}, \mathbf{A}, \mathbf{D}$ & 6 & 14 \\
\hline 70 & $32 / 02-1 \mathrm{~V}$ & $\mathbf{A}, \mathbf{D}, \mathbf{D}, \mathbf{D}$ & 1 & 102 \\
\hline 70 & $32 / 02-1 \mathrm{~V}$ & $\mathbf{B}, \mathbf{A}, \mathbf{B}, \mathbf{A}$ & 1 & 16 \\
\hline 70 & $32 / 02-1 \mathrm{~V}$ & $\mathbf{B}, \mathbf{B}, \mathbf{B}, \mathbf{D}$ & 2 & 11 \\
\hline 70 & $32 / 02-1 \mathrm{~V}$ & $\mathbf{B}, \mathbf{D}, \mathbf{B}, \mathbf{D}$ & 1 & 18 \\
\hline 70 & $32 / 02-1 \mathrm{~V}$ & $\mathbf{C}, \mathbf{B}, \mathbf{A}, \mathbf{B}$ & 1 & 4 \\
\hline 70 & $32 / 02-1 \mathrm{~V}$ & $\mathbf{C}, \mathbf{B}, \mathbf{C}, \mathbf{B}$ & 2 & 4 \\
\hline 70 & $32 / 02-1 \mathrm{~V}$ & $\mathbf{C}, \mathbf{C}, \mathbf{B}, \mathbf{B}$ & 1 & 1 \\
\hline 70 & $32 / 02-1 \mathrm{~V}$ & $\mathbf{C}, \mathbf{D}, \mathbf{A}, \mathbf{D}$ & 6 & 28 \\
\hline 70 & $32 / 02-1 V$ & $\mathbf{C}, \mathbf{D}, \mathbf{D}, \mathbf{D}$ & 1 & 67 \\
\hline
\end{tabular}

Aparece un hablante con bastantes características poco frecuentes en la base de datos (presenta ocho marcadores en los que el valor «intermd» es inferior a 50) y muchas características muy frecuentes en la base de datos que se repiten también mucho en el hablante (así pasa con el valor 10 de «intramd» con un valor «intermd» de 93). El valor del coeficiente de dispersión del hablante para estos datos es 2,329 respecto de un valor global de 0,871 .

Analicemos en detalle los marcadores de presencia más extremos del hablante $\mathrm{n}^{\mathrm{o}} 70$. Por una parte aquellos más identificativos por ser menos frecuentes en el total de la base de datos y, por otra, los más frecuentes que, consiguientemente, resultan menos individualizadores: 


\begin{tabular}{|c|c|c|}
\hline MD & Intramd / intermd & \multirow{2}{*}{$\begin{array}{l}\text { Este marcador se corresponde } \\
\text { con } / \mathrm{R} / \text {, en realización asimilada } \\
\text { a } / 1 / \text {, dos mediciones y relevante } \\
\text { acumulación de energía. }\end{array}$} \\
\hline $\mathrm{C}, \mathrm{C}, \mathrm{B}, \mathrm{B}$ & $(1 / 1)$ & \\
\hline MD & Intramd / intermd & \multirow{2}{*}{$\begin{array}{l}\text { Este marcador se corresponde } \\
\text { con /N/, acumulación de energía } \\
\text { armónica, una medición } \\
\text { y relevante duración. }\end{array}$} \\
\hline C, B, A, B & $(1 / 4)$ & \\
\hline MD & Intramd / intermd & \multirow{2}{*}{$\begin{array}{l}\text { Este marcador se corresponde } \\
\text { con /s/, en realización aspirada, } \\
\text { sin medición y sin valoración. }\end{array}$} \\
\hline $\mathrm{A}, \mathrm{D}, \mathrm{D}, \mathrm{D}$ & $(1 / 102)$ & \\
\hline MD & Intramd / intermd & \multirow{3}{*}{$\begin{array}{l}\text { Este marcador se corresponde } \\
\text { con } / \mathrm{i} /, / \mathrm{e} /, / \mathrm{a} / \mathrm{:} \\
\text { 1. Tres marcadores distintivos } \\
\text { con } / \mathrm{i} /, \text { altura formántica, } \\
\text { dos mediciones y realización } \\
\text { retrasada. } \\
\text { 2. Un marcador distintivo con } \\
\text { /e/, altura formántica, } \\
\text { dos mediciones y realización } \\
\text { retrasada. } \\
\text { 3. Seis marcadores distintivos } \\
\text { con /a/, altura formántica, } \\
\text { dos mediciones y realización } \\
\text { retrasada. }\end{array}$} \\
\hline $\mathrm{A}, \mathrm{A}, \mathrm{B}, \mathrm{A}$ & $(10 / 93)$ & \\
\hline & & \\
\hline & & \\
\hline
\end{tabular}

Haciendo una valoración de estos datos, puede apreciarse que los dos primeros marcadores son altamente individualizadores, a la vista de los valores de «intermd»e «intramd». Por otro lado, el marcador de mayor valor «intramd» $(10)$, resulta tener un valor medio de «intermd» (93) y representa un alto porcentaje de ese valor medio (el 9,3\% de 93) siendo además un marcador distintivo de alto valor cualitativo (A, A, B, A). 
2. Información resumida del expediente 128/99-2V correspondiente al Hablante $n^{\circ} 27$

\begin{tabular}{|c|c|c|c|c|}
\hline habla & exp & md & intramd & intermd \\
\hline 27 & $128 / 99-2 V$ & $\mathbf{A}, \mathbf{A}, \mathbf{A}, \mathbf{A}$ & 4 & 180 \\
\hline 27 & $128 / 99-2 \mathrm{~V}$ & $\mathbf{A}, \mathbf{A}, \mathbf{C}, \mathbf{D}$ & 1 & 45 \\
\hline 27 & $128 / 99-2 \mathrm{~V}$ & $\mathbf{B}, \mathbf{A}, \mathbf{A}, \mathbf{A}$ & 2 & 38 \\
\hline 27 & $128 / 99-2 \mathrm{~V}$ & $\mathbf{B}, \mathbf{D}, \mathbf{A}, \mathbf{D}$ & 2 & 121 \\
\hline 27 & $128 / 99-2 \mathrm{~V}$ & $\mathbf{C}, \mathbf{C}, \mathbf{D}, \mathbf{D}$ & 2 & 145 \\
\hline
\end{tabular}

Este hablante cuenta con muy pocas características particulares, pues sólo dos de los cinco marcadores tienen valores de «intermd» menores a 50, lo que indica características con valores muy frecuentes en la base de datos, y resta singularidad a las locuciones analizadas. A su vez, el marcador con valor más alto de «intramd» resulta ser precisamente el correspondiente más alto para «intermd». El valor del coeficiente de dispersión del hablante para estos datos es 0,307 y el valor global es 0,871 .

Si desarrollamos el análisis de los marcadores de presencia más extremos del hablante $\mathrm{n}^{\mathrm{o}}$ 27, encontramos:

\begin{tabular}{|c|c|c|}
\hline MD & Intramd / intermd & \multirow{3}{*}{$\begin{array}{l}\text { Este marcador se corresponde } \\
\text { con: } \\
\text { 1. Dos realizaciones de /a/, altura } \\
\text { formántica, una medición } \\
\text { y realización adelantada. } \\
\text { 2. Dos realizaciones de /i/, altura } \\
\text { formántica, una medición } \\
\text { y realización adelantada. }\end{array}$} \\
\hline $\mathrm{A}, \mathrm{A}, \mathrm{A}, \mathrm{A}$ & $(4 / 180)$ & \\
\hline & & \\
\hline MD & Intramd / intermd & \multirow{2}{*}{$\begin{array}{l}\text { Este marcador se corresponde } \\
\text { con dos realizaciones de /o/, } \\
\text { altura formántica, una medición } \\
\text { y realización adelantada. }\end{array}$} \\
\hline $\mathrm{B}, \mathrm{A}, \mathrm{A}, \mathrm{A}$ & $(2 / 38)$ & \\
\hline
\end{tabular}


También puede resultar interesante analizar un caso cuyo coeficiente de dispersión se encuentra, relativamente próximo al valor global:

\section{Información resumida del expediente 71/02-2V correspondiente al Hablante $n^{\circ} 105$}

El hablante 105 con expediente 71/02-2V, a través del resumen de la información obtenida, presenta la mitad de características particulares, pues seis de los doce marcadores tienen valores de «intermd» menores a 50, señalando características con valores muy poco frecuentes en la base de datos aportando, por tanto, singularidad al hablante. Sin embargo, la otra mitad de marcadores indica precisamente todo lo contrario.

\begin{tabular}{|c|c|c|c|c|}
\hline habla & exp & md & intramd & intermd \\
\hline 105 & $71 / 02-2 \mathrm{~V}$ & A, B, D, C & 5 & 5 \\
\hline 105 & $71 / 02-2 \mathrm{~V}$ & D, C, A, D & 1 & 7 \\
\hline 105 & $71 / 02-2 \mathrm{~V}$ & B, C, B, D & 1 & 14 \\
\hline 105 & $71 / 02-2 \mathrm{~V}$ & $\mathrm{D}, \mathrm{B}, \mathrm{B}, \mathrm{D}$ & 1 & 23 \\
\hline 105 & $71 / 02-2 \mathrm{~V}$ & $\mathrm{~B}, \mathrm{~B}, \mathrm{~A}, \mathrm{D}$ & 1 & 31 \\
\hline 105 & $71 / 02-2 \mathrm{~V}$ & $\mathrm{~B}, \mathrm{D}, \mathrm{A}, \mathrm{C}$ & 1 & 35 \\
\hline 105 & $71 / 02-2 \mathrm{~V}$ & $\mathrm{C}, \mathrm{B}, \mathrm{B}, \mathrm{D}$ & 1 & 55 \\
\hline 105 & $71 / 02-2 \mathrm{~V}$ & B, C, A, D & 1 & 77 \\
\hline 105 & $71 / 02-2 \mathrm{~V}$ & A, C, A, D & 2 & 84 \\
\hline 105 & $71 / 02-2 \mathrm{~V}$ & A, A, B, D & 4 & 93 \\
\hline 105 & $71 / 02-2 \mathrm{~V}$ & A, C, D, D & 4 & 101 \\
\hline 105 & $71 / 02-2 \mathrm{~V}$ & C, C, D, D & 10 & 145 \\
\hline
\end{tabular}

El valor del coeficiente de dispersión del hablante para estos datos es 0,627, muy próximo al valor global $(0,871)$, aunque algo más bajo. Para más detalle, puede analizarse la tabla desglosada de sus marcadores distintivos: 


\begin{tabular}{|c|c|c|c|c|c|c|c|c|c|c|c|c|c|c|c|}
\hline HABLA & EXPTE & SEXO & FYM & CODIU & CODIP & CODIV & QCL_1 & QCL_2 & QCL_3 & QCL_4 & $Q \mathrm{QU}$ & QP & $\mathrm{QY}$ & QV & mdabcd \\
\hline 105 & $71 / 02-2 \mathrm{~V}$ & 1 & 0 & 13 & 34 & 1 & 1 & 2 & 1 & 2 & C & C & $\mathrm{D}$ & D & C,C,D,D \\
\hline 105 & $71 / 02-2 \mathrm{~V}$ & 1 & 0 & 13 & 34 & 1 & 1 & 2 & 1 & 2 & C & C & D & D & C,C,D,D \\
\hline 105 & $71 / 02-2 \mathrm{~V}$ & 1 & 0 & 13 & 34 & 1 & 1 & 2 & 1 & 2 & C & C & $\mathrm{D}$ & D & $\mathrm{C}, \mathrm{C}, \mathrm{D}, \mathrm{D}$ \\
\hline 105 & $71 / 02-2 \mathrm{~V}$ & 1 & 0 & 22 & 5 & 1 & 1 & 2 & 1 & 2 & C & C & $\mathrm{D}$ & D & C, C,D,D \\
\hline 105 & $71 / 02-2 \mathrm{~V}$ & 1 & 0 & 22 & 5 & 1 & 1 & 2 & 1 & 2 & C & C & $\mathrm{D}$ & $\mathrm{D}$ & C,C,D,D \\
\hline 105 & $71 / 02-2 \mathrm{~V}$ & 1 & 0 & 22 & 16 & 1 & 1 & 2 & 1 & 2 & C & C & $\mathrm{D}$ & D & $\mathrm{C}, \mathrm{C}, \mathrm{D}, \mathrm{D}$ \\
\hline 105 & $71 / 02-2 \mathrm{~V}$ & 1 & 0 & 22 & 16 & 1 & 1 & 2 & 1 & 2 & $\mathrm{C}$ & C & $\mathrm{D}$ & D & C,C,D,D \\
\hline 105 & $71 / 02-2 \mathrm{~V}$ & 1 & 0 & 22 & 16 & 1 & 1 & 2 & 1 & 2 & C & C & D & D & C,C,D,D \\
\hline 105 & $71 / 02-2 \mathrm{~V}$ & 1 & 0 & 22 & 16 & 1 & 1 & 2 & 1 & 2 & C & C & D & D & C,C,D,D \\
\hline 105 & $71 / 02-2 \mathrm{~V}$ & 1 & 0 & 22 & 16 & 1 & 1 & 2 & 1 & 2 & C & C & $\mathrm{D}$ & D & C,C,D,D \\
\hline 105 & $71 / 02-2 \mathrm{~V}$ & 1 & 0 & 24 & 16 & 1 & 1 & 2 & 1 & 4 & A & C & D & D & A,C,D,D \\
\hline 105 & $71 / 02-2 \mathrm{~V}$ & 1 & 0 & 24 & 16 & 1 & 1 & 2 & 1 & 4 & A & C & $\mathrm{D}$ & D & A,C,D,D \\
\hline 105 & $71 / 02-2 \mathrm{~V}$ & 1 & 0 & 24 & 16 & 1 & 1 & 2 & 1 & 4 & A & C & D & D & $\mathrm{A}, \mathrm{C}, \mathrm{D}, \mathrm{D}$ \\
\hline 105 & $71 / 02-2 \mathrm{~V}$ & 1 & 0 & 24 & 16 & 1 & 1 & 2 & 1 & 4 & A & C & $\mathrm{D}$ & D & $\mathrm{A}, \mathrm{C}, \mathrm{D}, \mathrm{D}$ \\
\hline 105 & $71 / 02-2 \mathrm{~V}$ & 1 & 2 & 15 & 2 & 1 & 1 & 3 & 3 & 3 & D & B & B & D & $\mathrm{D}, \mathrm{B}, \mathrm{B}, \mathrm{D}$ \\
\hline 105 & $71 / 02-2 \mathrm{~V}$ & 1 & 1 & 32 & 1 & 1 & 1 & 2 & 4 & 3 & D & C & A & D & $\mathrm{D}, \mathrm{C}, \mathrm{A}, \mathrm{D}$ \\
\hline 105 & $71 / 02-2 \mathrm{~V}$ & 1 & 2 & 5 & 2 & 1 & 1 & 3 & 3 & 2 & C & B & B & D & $\mathrm{C}, \mathrm{B}, \mathrm{B}, \mathrm{D}$ \\
\hline 105 & $71 / 02-2 \mathrm{~V}$ & 1 & 0 & 24 & 30 & 19 & 3 & 3 & 1 & 4 & A & B & $\mathrm{D}$ & C & $\mathrm{A}, \mathrm{B}, \mathrm{D}, \mathrm{C}$ \\
\hline 105 & $71 / 02-2 \mathrm{~V}$ & 1 & 0 & 24 & 30 & 19 & 3 & 3 & 1 & 4 & A & B & $\mathrm{D}$ & C & $\mathrm{A}, \mathrm{B}, \mathrm{D}, \mathrm{C}$ \\
\hline 105 & $71 / 02-2 \mathrm{~V}$ & 1 & 0 & 24 & 30 & 19 & 3 & 3 & 1 & 4 & A & B & $\mathrm{D}$ & C & $A, B, D, C$ \\
\hline 105 & $71 / 02-2 \mathrm{~V}$ & 1 & 0 & 24 & 30 & 19 & 3 & 3 & 1 & 4 & A & B & $\mathrm{D}$ & C & $A, B, D, C$ \\
\hline 105 & $71 / 02-2 \mathrm{~V}$ & 1 & 0 & 24 & 30 & 19 & 3 & 3 & 1 & 4 & A & B & $\mathrm{D}$ & C & $A, B, D, C$ \\
\hline 105 & $71 / 02-2 \mathrm{~V}$ & 1 & 2 & 12 & 1 & 1 & 1 & 2 & 3 & 1 & B & C & B & D & $\mathrm{B}, \mathrm{C}, \mathrm{B}, \mathrm{D}$ \\
\hline 105 & $71 / 02-2 \mathrm{~V}$ & 1 & 1 & 29 & 21 & 1 & 1 & 2 & 4 & 1 & B & C & $\mathrm{A}$ & D & $\mathrm{B}, \mathrm{C}, \mathrm{A}, \mathrm{D}$ \\
\hline 105 & $71 / 02-2 \mathrm{~V}$ & 1 & 1 & 29 & 13 & 1 & 1 & 3 & 4 & 1 & B & B & A & D & $\mathrm{B}, \mathrm{B}, \mathrm{A}, \mathrm{D}$ \\
\hline 105 & $71 / 02-2 \mathrm{~V}$ & 1 & 1 & 12 & 39 & 21 & 3 & 1 & 4 & 1 & B & D & A & C & $\mathrm{B}, \mathrm{D}, \mathrm{A}, \mathrm{C}$ \\
\hline 105 & $71 / 02-2 \mathrm{~V}$ & 1 & 1 & 24 & 21 & 1 & 1 & 2 & 4 & 4 & A & C & $\mathrm{A}$ & D & $\mathrm{A}, \mathrm{C}, \mathrm{A}, \mathrm{D}$ \\
\hline 105 & $71 / 02-2 \mathrm{~V}$ & 1 & 1 & 24 & 21 & 1 & 1 & 2 & 4 & 4 & A & C & A & D & $\mathrm{A}, \mathrm{C}, \mathrm{A}, \mathrm{D}$ \\
\hline 105 & $71 / 02-2 \mathrm{~V}$ & 1 & 2 & 7 & 3 & 1 & 1 & 4 & 3 & 4 & A & A & B & D & $\mathrm{A}, \mathrm{A}, \mathrm{B}, \mathrm{D}$ \\
\hline 105 & $71 / 02-2 \mathrm{~V}$ & 1 & 2 & 7 & 3 & 1 & 1 & 4 & 3 & 4 & A & A & B & D & $\mathrm{A}, \mathrm{A}, \mathrm{B}, \mathrm{D}$ \\
\hline 105 & $71 / 02-2 \mathrm{~V}$ & 1 & 2 & 2 & 3 & 1 & 1 & 4 & 3 & 4 & A & A & B & D & $A, A, B, D$ \\
\hline 105 & $71 / 02-2 \mathrm{~V}$ & 1 & 2 & 2 & 3 & 1 & 1 & 4 & 3 & 4 & A & A & B & D & $A, A, B, D$ \\
\hline
\end{tabular}


Si se realiza un análisis desarrollado de los marcadores de presencia más extremos del hablante, se observarán aquellos más identificativos, por ser menos frecuentes en el total de la base de datos, así como los más frecuentes que, a su vez, resultan ser los menos individualizadores:

\begin{tabular}{|c|c|c|}
\hline MD & Intramd / intermd & \multirow{2}{*}{$\begin{array}{l}\text { Este marcador se corresponde } \\
\text { con } / \mathrm{R} / \text {, realización fonética } \\
\text { cero, sin mediciones y sin } \\
\text { valoración. }\end{array}$} \\
\hline $\mathrm{A}, \mathrm{B}, \mathrm{D}, \mathrm{C}$ & $(5 / 5)$ & \\
\hline MD & Intramd / intermd & \multirow{2}{*}{$\begin{array}{l}\text { Este marcador se corresponde } \\
\text { con } / \hat{\jmath} / \text {, acumulación de energía, } \\
\text { una medición y sin valorar. }\end{array}$} \\
\hline $\mathrm{D}, \mathrm{C}, \mathrm{A}, \mathrm{D}$ & $(1 / 7)$ & \\
\hline MD & Intramd / intermd & \multirow{3}{*}{$\begin{array}{l}\text { Este marcador se corresponde } \\
\text { con } / \mathrm{l} / \mathrm{y} / \mathrm{R} / \text { : } \\
\text { 1. Cinco marcadores distintivos } \\
\text { con } / \mathrm{R} /, \text { realización fonética } \\
\text { cero, sin medición y sin valorar } \\
\text { 2. Dos marcadores distintivos } \\
\text { con } / \mathrm{R} /, \text { asimilación regresiva, } \\
\text { sin medición y sin valorar. } \\
\text { 3. Tres marcadores distintivos } \\
\text { con } / 1 /, \text { sustitución por el } \\
\text { fonema } / \mathrm{r} /, \text { sin mediciones } \\
\text { y sin valorar. }\end{array}$} \\
\hline C, C, D, D & $(10 / 145)$ & \\
\hline & & \\
\hline
\end{tabular}

\section{RESUMEN DE LOS RESULTADOS}

El presente estudio ha permitido establecer una jerarquía de distintos parámetros acústicos y fono-articulatorios del español hablado, en razón a su peso identificativo. En una primera etapa, los parámetros objeto de estudio han sido cuantificados y clasificados en cuatro tablas de resultados perfectamente diferenciadas. Posteriormente, el tratamiento conjunto de estas cuatro tablas ha introducido la definición de dos nuevos conceptos: «marcador distintivo»y «marcador de presencia».

La información correspondiente a cada hablante ha sido distribuida en un número variable de «marcadores distintivos», identificados me- 
diante ítems nominales con sentido cualitativo. Estos marcadores representan la información que en cada caso utiliza el experto como elemento de comparación. Pueden repetirse en un mismo hablante (intravariabilidad) y muestran una mayor o menor redundancia en la población de referencia (intervariabilidad). Los valores de frecuencia asociados a dichos conceptos, en cada hablante, completan el nivel de relevancia identificativa del marcador, que así considerado constituye el «marcador de presencia».

La evaluación de los marcadores de presencia de cada hablante confiere el carácter individualizador de cada comparación que realiza el experto, tanto desde una perspectiva intrapersonal como interpersonal. De esta forma, aquellas características poco frecuentes en la población de referencia y muy frecuentes en el hablante confieren un alto valor identificativo, mientras que aquellas muy habituales en la población de referencia, denotan un escaso valor identificativo.

El mayor o menor número de marcadores de presencia no resulta significativo respecto del valor de identificación de cada hablante (coeficiente de dispersión) y sí la relevancia obtenida del conjunto de marcadores en el estudio del hablante. Es decir, como parece lógico, no es tan importante contar con muchos marcadores sino contar con un número suficiente de aquellos que aporten datos relevantes.

Por último, se establece una referencia de tipicidad por hablante, derivada del cálculo de la razón entre su carácter intrapersonal e interpersonal. Dicha referencia enmarca y asocia cada uno de los locutores con el resto de los que integran la población utilizada.

\section{Conclusiones}

Cuando se efectúa una comparación de actos de habla en entornos forenses, la evaluación de los rasgos identificativos viene influenciada por cuatro factores fundamentales: la propia naturaleza de las muestras objeto de estudio, la metodología utilizada, la cualificación y experiencia del científico evaluador y los parámetros que dimensionan las poblaciones de referencia. Con independencia de los sistemas de análisis desarrollados, las referencias poblacionales referidas a los distintos parámetros condicionan el carácter individualizador de los mismos. De ahí la gran importancia de fomentar líneas de investigación para establecer distribuciones poblacionales de las diferentes realizaciones del habla, que permitan dimensionar correctamente el peso identificativo de cada elemento analizado.

Al margen de la estratificación de parámetros alcanzada en este estudio, resulta necesario subrayar un importantísimo aspecto que igualmente es puesto de manifiesto. La relevancia identificativa de un rasgo 
acústico o fono-articulatorio no sólo ha de asociarse a las características de una población determinada; a su vez, cada escenario de comparación debe ser supervisado por el experto en cada una de sus distintas fases (admisión y selección de muestras, elección de opciones de análisis, normalización e interpretación de resultados). Las poblaciones de referencia -entendidas con carácter general y no en su estricto sentido estadístico-, aunque necesarias, han de considerarse orientativas pues simplemente representan referencias de actos de habla emitidos y registrados en contextos concretos. Lo mismo puede decirse y referirse de las muestras objeto de cotejo ${ }^{1}$.

\section{REFERENCIAS BIBLIOGRÁFICAS}

Battaner, E.; Gil, J.; Marrero, V.; Llisterri, J.; Carbó, C.; Machuca, M. J.; De LA Mota, C. y Ríos, A. (2003): «Estudio acústico de la variación inter e intralocutor en español (VILE)», Actas del II Congreso de la Sociedad Española de Acústica Forense (SEAF), Barcelona 10-11 abril 2003, pp. 59-70.

Black, J. W.; Lashbrook, W.; Nash, W.; Oyer, H. J.; Pedrey, C.; Tosi, O. y Truby, H. (1973): «Replay to speaker identification by speech spectrograms: some further observations», JASA 54, pp. 535-537.

Bolt, R. H.; Cooper, F. S.; David, E. C.; Denes, P. B.; Pickett, J. M. y Stevens, K. N. (1970): «Speaker Identification by Speech Spectrograms», Journal of the Acoustical Society of America 47, pp. 597-613.

BRAUN, A. (1995): «Fundamental frequency, how speaker-specific is it?», BEIPHOL 64: Studies in Forensic Phonetics, p. 15.

CAIn, S. (1999): Voice Comparison Standards, American Board of Recorded Evidence, AES, Inc. web site.

Clifford, B. R. (1980): «Voice identification by human listeners: on earwitness reliability», Law Human Behavior 4, pp. 373-394.

Delgado Romero, C. (2001): La identificación de locutores en el ámbito forense, Tesis doctoral, Universidad Complutense de Madrid, web site, pp. 212, 214-217 y 259-279.

DOHERTy, E. T. (1976): «An evaluation of selected acoustic parameters for use in speaker identification», Journal of Phonetics 4, pp. 321-326.

DOMMELEN, W. A. VAN (1997): «The contribution of speech rhythm and pitch to speaker recognition", Language and Speech 30, pp. 325-338.

ElaAd, E.; SegeV, S. y Tobin, Y. (1998): Long-term Working Memory in Voice Identification, Psychology, Crime \& Law, vol. 0, pp. 1-16.

ENDRESS, W.; BAMBACH, W. y FlÖSSER, G. (1971): «Voice Spectrograms as a function of age, voice disguise and voice imitation», JASA 49, pp. 1842-1848.

GIFI, A. (1990): Nonlinear multivariate analysis, Chichester, John Wiley and Sons.

\footnotetext{
${ }^{1}$ Queremos expresar nuestro agradecimiento a todos los compañeros del laboratorio de Acústica Forense de la Policía Científica de España, pues la elaboración del presente trabajo no hubiera sido posible sin su contribución.
} 
Gray, C. H. y Kopp, G. A. (1944): Voiceprint Identification, Bell Telephone Laboratories Report, Bell Laboratories, pp. 1, 3, 13, 14.

HaLl, M. C. (1975): Spectrografic Analysis of Interspeaker and Intraspeaker variables of professional Mimicry, Master Thesis, Michigan State University.

Hartigan, J. A. (1975): Clustering algorithms, Nueva York, John Wiley and Sons.

Hartmann, D. (1979): «The perceptual identity and characteristics of aging in normal male adult speakers», Journal of Communication Disorders 12, pp. 53-61.

HAZEN, B. M. (1973): «Effects of different phonetics contexts on spectrographic Speaker Identification", JASA 54, pp. 650-660.

Helfrich, H. (1979): Age markers in speech, Eds. Sherer/Giles, pp. 63-107.

Hollien, H. (1990): The acoustics of crime, Nueva York, Plenum Press, pp. 197-198.

Houlihan, K. (1979): The Effects of Disguise on Speaker Identification from Sound Spectrograms, Ámsterdam, Current issues in Phonetic Sciences, J. Benjamins, B. V., pp. 811-820.

KerSTA, L. G. (1962): «Voiceprint Identification», Nature 196, pp. 1253-1257.

KoENIG, B. E. (1986): «Spectrographic voice identification: a forensic survey, Letter to the editor», Journal of the Acoustical Society of America 79, pp. 20882090.

KoENIG, B. E. (1991): «Voice comparison standards», Journal of Forensic identification 41, 5, International association for Identification, pp. 384-387.

Köster, P. (1981): Auditive Sprechererkenung bei Experten und Naiven, Hamburgo, Festschrift Wrangler, Helmut Buske AG, 52, pp. 171-180.

Koval, S. y KRYNOv, S. (2000): Practice of usage of spectral analysis for forensic speaker identification, San Petersburgo, Speech Technology Center [Sin referencias concretas de publicación. Divulgado por el Instituto a clientes comerciales].

Koval, S.; Ilyina, O. y Khitina, M. (1999): Practice of usage of additive and linguistic features for forensic Speaker Identification, San Petersburgo, Speech Technology Center [Sin referencias concretas de publicación. Divulgado por el Instituto a clientes comerciales].

Kuwabara, H. y Sagisaka, Y. (1995): «Acoustic characteristics of speaker individuality: control and conversion», Speech Communication 16, pp. 165-173.

KüNZEL, H. (1987): Sprechererkennung, Heidelberg, Kriminalistik Verlag GmbH, pp. 33-79.

LAdEFodged, P. y LAdEFodged, J. (1980): The ability of listeners to identify voices, Los Ángeles, UCLA Working Papers in Phonetics, 49, pp. 43-51.

Marrero, V.; Gil, J. y Battaner, E. (2003): «Inter-speaker Variation in Spanish. An Experimental and Acoustic Preliminary Approach», Proceedings of the $15^{\text {th }}$ International Congress of Phonetic Sciences, Barcelona, 3-9 august 2003, pp. 703-706.

MCGlone, R. E. y Hollien, H. (1963): «Vocal pitch characteristics of old women», JSHR 6, pp. 164-170.

Morris, R. J. y Brown, W. S. Jr. (1998): «Aged related differences in Fo and pitch sigma among females», The IASCP bulletin 1-2, pp. 36-39.

Neiman, G. y Applegate, J. (1990): «Accuracy of listener judgements of perceived age relative to chronological age in adults», Folia Phoniatrica 42, pp. 327-330. 
NOLAN, F. (1990): «The limitations of auditory-phonetic speaker identification, en Kniffka, H. (ed.), Texte zu Theorie und Praxis Forensischer Linguistik, Tubinga, Niemeyer, pp. 457-479.

Nolan, F. (1991): «Forensic Phonetics», Journal of Linguistics 27, Cambridge University Press,

Pollack, I.; Pickett, J. M. y Sumby, W. H. (1954): «On the identification of speakers by voice», Journal of the Acoustical Society of America 26, pp. 403-412.

Popov, H. F.; Linkov, A. N. y Baicharov, M. V. (1996): «Personal identification by Russian speech phonograms on the automatized system», en Fesenko A. F. (ed.), DIALECT: Manual for experts, Military Unit n. 34435, Moscú.

Potter, R.; Kopp, G. y GreEn, H. (1947): Visible Speech, Nueva York, Van Nostrand.

Quilis, A. (1981): Fonética acústica de la lengua española, Madrid, Ed. Gredos.

REICH, A. y DukE (1979): «Effects of selected vocal disguises upon speaker identification by listening», Journal of the Acoustical Society of America 66, p. 1023.

Rose, P. (2002): Forensic Speaker Identification, Londres, Taylor \& Francis Group, pp. 34-41.

Rothman, H. (1997): «A Perceptual (Aural) and Spectrographic Identification of Talkers with Similar Sounding Voices», Proceedings International Conference Crime Counter Measures, Oxford, pp. 37-42.

SMrkovski, L. (1976): «Study of speaker identification by aural and visual examination of non-contemporary speech samples», Journal of Official Analytical Chemists 59, pp. 927-931.

STEVENS, K. (1971): «Sources of inter and intra-speaker variability in the acoustic properties of speech sounds», en Charbonneau, R. y Rigault, A. (eds.), Proceedings of the $7^{\text {th }}$ International Congress of Phonetic Sciences, La Haya, Mouton, pp. 206-232.

Suzuki, T.; TAnimoto, M.; OsanaI, T.; Kido, H. y Kamada, T. (1997): «Speaker retrieval system for investigative operation", Paper presented in the $82^{\text {th }}$ Annual I.A.I. Conference, Danvers (Massachussets),

Tosi, O.; Oyer, H.; Lashbrook, W.; Pedrey, C.; Nicol, J. y NASH, E. (1972): «Experiment on voice identification", Journal of the Acoustical Society of America 51, pp. 2030-2043.

Tosi, O. y GreEnwald, M. (1978): «Voice identification by subjective methods of minority group voices», Presentación de la VII reunión de la I.A.V.I., Nueva Orleáns (Luisiana).

Tosi, O. (1979): Voice Identification. Theory and legal applications, Baltimore (Maryland), University Park Press, pp. 40, 114-115. 